\title{
Toward Proof of Lusztig's Conjecture Concerning Negative Level Representations of Affine Lie Algebras
}

\author{
SHRaWAN Kumar \\ School of Maths, T.I.F.R., Bombay 400005, India, and \\ The University of North Carolina, Chapel Hill, North Carolina 27599-3250 \\ Communicated by Leonard Scott \\ Received October 30, 1991
}

\section{INTRODUCTION}

Let $\stackrel{9}{\mathrm{~g}}$ be a finite dimensional simple Lie algebra over $\mathbb{C}$ and let $\mathfrak{g}=\operatorname{Aff} f^{\wedge}(\stackrel{g}{)})$ be the affine Lie algebra bidually associated to $\mathfrak{g}$ (cf. Definition 2.1). Let $h$ be the dual Coxeter number of $g$ (cf. Section 1.1), and let $l \geqslant 1$ be any integer. Lusztig made a conjecture determining the decomposition (in an appropriate Grothendieck group) of irreducible highest weight modules of $\mathrm{g}$ of level $-l-h$ (where the level is the scalar by which the canonical central element $c \in \mathfrak{g}$ acts) in terms of the Verma modules of the same level, which involves the value of the KL-polynomials for the affine Weyl group $W$ at 1 (cf. Conjecture 2.7). (Actually Lusztig explicitly wrote down this conjecture only for the case when $\dot{\mathfrak{g}}$ is simply-laced, in which case $g$ is nothing but the affine Lie algebra $\hat{L}(\mathfrak{g})$; cf. Section 1.2.) The aim of this paper is to show that the celebrated Kazhdan-Lusztig conjecture (which determines the decomposition of the irreducible module $L(-w \rho-\rho)$ in terms of the Verma modules $M(-v \rho-\rho))$ for the affine Lie algebra $g$ implies the validity of Lusztig's conjecture for every $l \geqslant 1$ (cf. Theorem 2.10). Of course the KL-conjecture is known in the finite case due to Beilinson-Bernstein and Brylinski-Kashiwara. Recently, L. Casian [C] has claimed to prove the KL-conjecture (for $L(-w \rho-\rho)$ ) in the affine case. Our work does not rely on Casian's work in any manner and would supplement his (or similar) results to establish the full Lusztig's conjecture concerning the negative level representations of affine Lie algebras.

The main ingredient in our proof is an extension of Jantzen's translation functor for the negative level representations of the affine Lie algebra $\mathfrak{g}$, developed in Section 1 of this paper. This functor is obtained by tensoring 
a negative level representation of $\mathfrak{g}$ with an integrable highest weight (irreducible) $g$-module and then projecting onto a component of certain specific type. This extension crucially relies on an earlier work of the author $[\mathrm{Ku}]$, concerning a vanishing result for the Ext functor corresponding to the negative level representations of $\mathfrak{g}$. Recall that the Jantzen's translation functor is studied in [DGK], for the positive level representations.

\section{Jantzen's Translation FunCtOR fOR NEgATIVE LEVEL REPRESENTATIONS}

(1.1) Notation. We follow the notation as in [K, Chap. 6].

Let $\mathfrak{g}=\mathfrak{g}(A)$ be the affine (possibly twisted affine) Kac-Moody Lie algebra over $\mathbb{C}$, associated to a generalized Cartan matrix $A=\left(a_{i j}\right)_{0 \leqslant i, j \leqslant n}$ of affine type. Let $\mathfrak{h} \subset \mathfrak{b}$ be the standard Cartan subalgebra, and the standard Borel subalgebra of $\mathfrak{g}$ (respectively). Observe that $\operatorname{dim}_{\mathbb{C}} \mathfrak{h}=n+2$. Let $\pi=\left\{\alpha_{0}, \ldots, \alpha_{n}\right\} \subset \mathfrak{h}^{*}$ be the set of simple roots (for $b$ ), and $\pi^{\vee}=\left\{\alpha_{0}^{\vee}, \ldots, \alpha_{n}^{\vee}\right\} \subset \mathfrak{h}$ the set of simple coroots. We denote by $Q$ (resp. $Q^{\vee}$ ) the root lattice $\sum_{i=0}^{n} \mathbb{Z} \alpha_{i} \subset \mathfrak{h}^{*}$ (resp. the coroot lattice $\left.\sum_{i=0}^{n} \mathbb{Z} \alpha_{i}^{\vee} \subset \mathfrak{h}\right)$. We define

$$
\mathfrak{h}_{\mathbb{Z}}^{*}=\left\{\lambda \in \mathfrak{h}^{*}: \lambda\left(\alpha_{i}^{\vee}\right) \in \mathbb{Z}, \text { for all } 0 \leqslant i \leqslant n\right\} .
$$

The Weyl group of $\mathrm{g}$ is denoted by $W$. It is a Coxeter group with simple reflections $\left\{s_{0}, \ldots, s_{n}\right\}$ as generators, where $s_{i}$ is the reflection corresponding to the simple root $\alpha_{i}$ (i.e., $s_{i} \lambda:=\lambda-\lambda\left(\alpha_{i}{ }^{v}\right) \alpha_{i}$, for all $\lambda \in \mathrm{b}^{*}$ ).

We fix an element $d \in \mathfrak{h}$ satisfying $\alpha_{i}(d)=0$ for $1 \leqslant i \leqslant n$, and $\alpha_{0}(d)=1$. Then

$$
\mathfrak{h}=\bigoplus_{i=0}^{n} \mathbb{C} \alpha_{i}^{\vee} \oplus \mathbb{C} d
$$

The centre of $\mathfrak{g}$ is one dimensional and is spanned by the canonical central element

$$
c:=\alpha_{0}^{\vee}+\sum_{i=1}^{n} a_{i}^{\vee} \alpha_{i}^{\vee} .
$$

We also define the imaginary root

$$
\delta:=\sum_{i=0}^{n} a_{i} \alpha_{i} \in Q
$$

where $a_{i}$ (resp. $a_{i}^{\vee}$ ) are the "labels" of the Dynkin diagram of $A$ (resp. the Dynkin diagram of the dual generalized Cartan matrix $A^{t}$ ) (cf. [K, tables 
in Chap.4]). The number $g:=\sum_{i=0}^{n} a_{i}$ (resp. the number $h:=$ $1+\sum_{i=1}^{n} a_{i}^{\vee}$ ) is called the Coxeter number of $\mathrm{g}$ (resp. the dual Coxeter number of $\mathfrak{g}$ ).

We fix an element $\rho \in \mathfrak{h}^{*}$ satisfying $\rho\left(\alpha_{i}^{v}\right)=1$, for all $0 \leqslant i \leqslant n$, and define the shifted action of $W$ on $\mathfrak{h}^{*}$ by

$$
w * \lambda=w(\lambda+\rho)-\rho, \quad \text { for any } \quad w \in W \text { and } \lambda \in \mathfrak{h}^{*} \text {. }
$$

Observe that the action $*$ does not depend upon the choice of $\rho$. Clearly $\rho(c)=h$.

(1.2) Untwisted Affine Algebras. To any finite dimensional simple Lie algebra $\stackrel{\mathrm{g}}{\mathrm{g}}$, there is associated the (untwisted) affine Kac-Moody Lie algebra $\mathfrak{g}=\hat{L}(\mathfrak{g}):=\mathfrak{g} \otimes_{\mathbb{C}} \mathbb{C}\left[t, t^{-1}\right] \oplus \mathbb{C} \oplus \oplus \mathbb{C} d$ (the Lie bracket structure in $\hat{L}(\stackrel{\mathfrak{g}}{)})$ is defined in [K, Chap. 7]), and this way we get all the untwisted affine Kac-Moody Lie algebras. The twisted ones are got from the untwisted ones by taking the fixed points of a finite order automorphism of $g$ coming

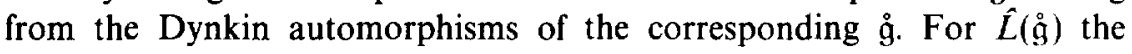
numbers $a_{i}$ 's are given by

$$
a_{0}=1, \quad \text { and } \quad \sum_{i=1}^{n} a_{i} \alpha_{i} \text { is the highest root for } \stackrel{9}{\mathrm{~g}} .
$$

(1.3) Verma Modules and the Relation $\sim$. Let $\mathfrak{g}, \mathfrak{b}$ be as in Section 1.1. For any $\lambda \in \mathfrak{h}^{*}$, we denote by $M(\lambda)$ the Verma module with highest weight $\lambda$, and $L(\lambda)$ is its irreducible quotient.

For any $\mathrm{g}$-module $M$, we say that $L(\lambda)$ is a constituent of $M$ if $L(\lambda)$ is a subquotient of $M$.

For any $\lambda, \mu \in \mathfrak{h}^{*}$, we call $\lambda \neg \mu$ if $L(\lambda)$ is a constituent of $M(\mu)$, and denote by $\sim$ the equivalence relation generated by $\rightarrow$.

Define a hyperplane $H$ of $\mathfrak{h}^{*}$ by $H=\left\{\lambda \in \mathfrak{h}^{*}:(\lambda+\rho)(c)=0\right\}$, where $\rho, c$ are as in Section 1.1. Then, as can be easily seen, $H=\left(\sum_{i=0}^{n} \mathbb{C} \alpha_{i}\right)-\rho$.

The following result will play a very crucial role in this paper.

(1.4) Theorem [Ku, Coro. 1.8 and Prop. 1.10]. Take any $\lambda \in K=$ $K^{w \cdot g}:=\mathfrak{h}^{*} \backslash H$. Then any $\mu \sim \lambda$ belongs to $K$. In fact $\mu \in W * \lambda$, where * is the shifted action as in Section 1.1. Conversely, for any $\lambda \in \mathfrak{h}_{\mathbb{Z}}^{*} \cap K$ (cf. Section 1.1) and any $w \in W, w * \lambda \sim \lambda$. More generally, for any $\lambda \in K$, $\mu \sim \lambda$ if and only if $\mu \in W(\lambda) * \lambda$, where $W(\lambda)$ is as defined in [Ku, Coro. 1.8].

(1.5) Decomposition of the Category $\mathcal{O}$. Let us begin by recalling the definition of the $B G G$ category $\mathcal{C}$, say from [DGK, Sect. 3]. The objects 
of this category are all those $\mathrm{g}$-modules $M$, which satisfy the following conditions:

(1) The module $M$ is $\mathfrak{h}$-semisimple, with finite dimensional weight spaces.

(2) There exist finitely many elements $\mu_{1}, \ldots, \mu_{k} \in \mathfrak{h}^{*}$, such that any weight $\mu$ of $M$ belongs to some $D\left(\mu_{i}\right)$, where

$$
D\left(\mu_{i}\right):=\mu_{i}-\sum_{i=0}^{n} \mathbb{Z}^{+} \alpha_{i}
$$

where $\mathbb{Z}^{+}:=\{0,1,2, \ldots\}$.

The morphisms in $\mathcal{O}$ are, by definition, all the g-module homomorphisms.

It is easy to see that for $M_{1}, M_{2} \in \mathcal{O}$, their tensor product $M_{1} \otimes M_{2}$ is again in the category $\mathcal{O}$.

For any equivalence class (w.r.t. $\sim$ ) $\wedge \subset \mathfrak{h}^{*}$, by $\mathscr{O}_{\wedge}$ we mean the full subcategory of $\mathcal{O}$ consisting of all the modules $M \in \mathcal{C}$ such that every constituent of $M$ has highest weight $\in \wedge$.

By [DGK, Thm. 4.2], any $M \in \mathcal{O}$ admits a decomposition

$$
M \approx\left(\oplus_{\wedge} M_{\wedge}\right) \oplus N
$$

where the sum runs over all the equivalence classes $\wedge$ of $K$ (under $\sim$ ), the submodule $M_{\wedge} \in \mathcal{O}_{\wedge}$, and all the constituents of $N$ have highest weights $\in H$ (cf. Theorem 1.4).

(1.6) Translation Functor. Fix a dominant integral weight $\theta \in \mathfrak{h}^{*}$ (i.e., $\theta\left(\alpha_{i}^{\vee}\right) \in \mathbb{Z}^{+}$, for all $\left.0 \leqslant i \leqslant n\right)$ and let $L(\theta)$ be the corresponding irreducible $\mathrm{g}$-module (which is known to be integrable, i.e., the g-action "exponentiates" to give an action of the corresponding affine Kac-Moody group).

For any $\lambda \in K$ such that $\lambda+\theta \in K$, we define the translation functor (extension of Jantzen's translation functor)

$$
T=T(\lambda, \theta): \mathscr{O}_{\wedge(i)} \rightarrow \mathcal{O}_{\wedge(\lambda+\theta)}, \quad \text { by } \quad T(M)=[M \otimes L(\theta)]_{\wedge(\lambda+\theta)},
$$

where $\wedge(\lambda)$ denotes the equivalence class of $\lambda$, and []$_{\wedge(\lambda+\theta)}$ denotes the $\wedge(\lambda+\theta)$-th component as guaranteed by $\left(I_{3}\right)$. (For $\lambda \in$ Tit's cone, the functor $T$ was defined in [DGK].)

Define the subset $D_{-} \subset \mathfrak{h}^{*}$ by

$$
\begin{aligned}
& D_{-}=\left\{\lambda \in \mathfrak{h}_{\mathbb{Z}}^{*}: \lambda\left(\alpha_{i}^{\vee}\right) \leqslant-1, \text { for all } 0 \leqslant i \leqslant n,\right. \text { and } \\
&\text { moreover } \left.\lambda\left(\alpha_{i}^{\vee}\right)<-1, \text { for at least one } i\right\} .
\end{aligned}
$$


Observe that any $\lambda$ such that $\lambda+\theta \in D_{-}$has the property that $\lambda$ and $\lambda+\theta$ both belong to $K$.

For any $\lambda \in \mathfrak{h}^{*}$ define the stabilizer $W_{\lambda}=\{w \in W: w \lambda=\lambda\}$.

(1.7) Proposition. Fix a dominant integral weight $\theta$. Then

(a) $T=T(\lambda, \theta): \mathcal{O}_{\wedge(\lambda)} \rightarrow \mathcal{O}_{\wedge(i+\theta)}$ is an exact functor for any $\lambda \in K$ such that $\lambda+\theta \in K$.

(b) For any $\lambda \in \mathfrak{h}^{*}$ such that $\lambda+\theta \in D_{-}$and any $w \in W$, the following is satisfied:

$\left(\mathrm{b}_{1}\right) \quad T(M(w * \lambda))=M(w *(\lambda+\theta))$,

$\left(\mathrm{b}_{2}\right) \quad T(L(w * \lambda))=L(w *(\lambda+\theta))$, if $w$ is the shortest element in the coset $w W_{i+\theta+\rho}$, and

$\left(b_{3}\right)$ Assume that $-(2 \rho+\lambda)$ is dominant (integral). Then $T(L(w * \lambda))=0$, if $w$ is not the shortest element in the coset $w W_{\lambda+\theta+\rho}$.

(1.8) Remark. Observe that for $\lambda+\theta \in D_{-}, W_{\lambda+\theta+\rho}$ is the subgroup of $W$ generated by the simple reflections it contains (see [K, Prop. 3.12]).

(1.9) Proof (of Proposition 1.7). (a) is clear because of $\left(\mathrm{I}_{3}\right)$.

Proof of $\left(\mathrm{b}_{1}\right)$. Let $\beta \in \mathfrak{h}^{*}$ be such that the $\beta$ th weight space of $L(\theta)$ is non-zero. We claim that $w * \lambda+\beta \in W *(\lambda+\theta)$ if and only if $\beta=w \theta$. (In particular the $\beta$ th weight space is one dimensional.):

Write $w * \lambda+\beta=v *(\lambda+\theta)$, for some $v \in W$. Then $v^{-1} w(\lambda+\rho)-(\lambda+\rho)=$ $\theta-v^{-1} \beta$, i.e. (putting $\lambda+\rho=-\mu$ ), $\mu-\theta=v^{-1} w\left(\mu-\theta+\theta-w^{-1} \beta\right.$ ), which gives

$$
\|\mu-\theta\|^{2}=\|\mu-\theta\|^{2}+\left\|\theta-w^{-1} \beta\right\|^{2}+2\left\langle\mu-\theta, \theta-w^{-1} \beta\right\rangle .
$$

By assumption $\mu-\theta$ is dominant and is non-zero and of course $\theta-w^{-1} \beta \epsilon$ $Q^{+}:=\sum_{i=0}^{n} \mathbb{Z}^{+} \alpha_{i}$, hence $\left\langle\mu-\theta, \theta-w^{-1} \beta\right\rangle \geqslant 0$. Further the Killing form $\langle$,$\rangle restricted to \sum_{i=0}^{n} \mathbb{R} \alpha_{i}$ is positive semi-definite, and moreover $\langle\beta, \beta\rangle=0$, for $\beta \in \sum_{i=0}^{n} \mathbb{R} \alpha_{i}$, if and only if $\beta \in \mathbb{R} \delta$ (see [K, Section 6.2]). In particular, for $(*)$ to hold, we should have $\theta-w^{-1} \beta \in \mathbb{R} \delta$. But since $\langle\mu-\theta, \delta\rangle>0,(*)$ is possible only if $\theta=w^{-1} \beta$. This establishes the claim.

Now $\left(b_{1}\right)$ follows from [DGK, Lemma 5.8].

Proof of $\left(b_{2}\right)$. By an argument identical to [DGK, p. 113, (b)], we conclude that $T(L(w * \lambda))$ is either 0 or else is isomorphic with $L(w *(\lambda+\theta))$. So we need only show that $T(L(w * \lambda)) \neq 0$ :

Observe that $M(w * \lambda)$ is a module of finite length, since $\lambda \in D_{-}$. In the Grothendieck group of $U(\mathfrak{g})$-modules, write

$$
M(w * \lambda)=\left(\sum_{i} n_{i} L(v * \lambda)\right)+L(w * \lambda), \quad \text { for some } n_{i^{\prime}} \in \mathbb{Z}^{+},
$$


where the sum runs over those elements $\bar{v}=v \bmod W_{\lambda+\rho} \in W / W_{\lambda+\rho}$ such that

$$
w * \lambda-v * \lambda \in Q^{+} \text {and is non-zero. }
$$

By the (a) and $\left(b_{1}\right)$ parts of this proposition, we get by applying $T$ to $\left(I_{4}\right)$,

$$
M(w *(\lambda+\theta))=\sum_{\bar{v}} n_{\bar{v}} T(L(v * \lambda))+T(L(w * \lambda)) .
$$

But if non-zero, $T(L(v * \lambda))=L(v *(\lambda+\theta))$, for any $v \in W$. In particular, if $T(L(w * \lambda))=0$, we get that $w *(\lambda+\theta)=v *(\lambda+\theta)$, for some $v \in W$ satisfying $\left(\mathrm{I}_{5}\right)$. But since $w$ is the shortest element in the coset $w W_{\lambda+\theta+\rho}$ (by assumption), $v \geqslant w$. But then $v * \lambda-w * \lambda \in Q^{+}$(since $\left.\lambda \in D_{-}\right)$. This contradicts $\left(\mathrm{I}_{5}\right)$, thereby proving that $T(L(w * \lambda))=L(w *(\lambda+\theta))$.

Proof of $\left(\mathrm{b}_{3}\right)$. By assumption $W_{\lambda+\rho}=(e)$. Write

$$
M(w * \lambda)=\left(\sum_{v \neq w} n_{v} L(v * \lambda)\right)+L(w * \lambda), \quad \text { for some } n_{v} \in \mathbb{Z}^{+} . \quad\left(I_{7}\right)
$$

Let $w_{1}$ be the shortest element in the coset $w W_{\lambda+\theta+\rho}$. Then, of course, $w$ admits a reduced decomposition $w_{1} \cdot s$, for some $s \in W_{j+\theta+\rho}$. Write $s=s_{i_{1}} \cdots s_{i_{k}}$ as a reduced decomposition, where $s_{i_{j}}$ 's are simple reflections contained in $W_{\lambda+\theta+\rho}$ (see Remark 1.8). It is easy to see that there is an embedding $M\left(\left(w_{1} s_{i_{1}} \cdots s_{i_{j}}\right) * \lambda\right) \zeta M\left(\left(w_{1} s_{i_{1}} \cdots s_{i_{j+1}}\right) * \lambda\right)$ for all $0 \leqslant j<k$. In particular, $L\left(w_{1} * \lambda\right)$ is a constituent of $M(w * \lambda)$, i.e., $n_{w_{1}} \neq 0$ in $\left(\mathbf{I}_{7}\right)$.

Applying the functor $T$ to $\left(I_{7}\right)$, we get

$$
M(w *(\lambda+\theta))=\sum_{v \neq w_{1}, w} n_{v} T(L(v * \lambda))+n_{w_{1}} T\left(L\left(w_{1} * \lambda\right)\right)+T(L(w * \lambda)),
$$
i.e.,

$$
\begin{aligned}
M(w *(\lambda+\theta))= & \sum_{v \neq w_{1}, w} n_{v} T(L(v * \lambda)) \\
& +n_{w^{\prime} 1} L\left(w_{1} *(\lambda+\theta)\right)+T(L(w * \lambda)),
\end{aligned}
$$

by $\left(\mathrm{b}_{2}\right)$-part of the proposition. But since the multiplicity of $L(w *(\lambda+\theta))$ in $M(w *(\lambda+\theta))$ is exactly 1 and $T(L(w * \lambda))$ is either $L(w *(\lambda+\theta))$ or 0 , we are forced to have $T(L(w * \lambda))=0$ (by $\left.\mathrm{I}_{8}\right)$. This proves the proposition. 
(1.10) Corollary. For any $\lambda \in D_{-}$, and $v, w \in W$ such that $v$ is the shortest element in its coset $v W_{\lambda+p}$, we have

$$
[M(w * \lambda), L(v * \lambda)]=[M(w *(-2 \rho)), L(v *(-2 \rho))],
$$

where $[$,$] denotes the multiplicity.$

Proof. For any $0 \leqslant i \leqslant n$, let $\rho_{i}$ denote (any) fundamental weight defined by $\rho_{i}\left(\alpha_{j}^{v}\right)=\delta_{i, j}$, for any $0 \leqslant j \leqslant n$. For a subset $S \subset\{0, \ldots, n\}$, let $\rho_{S}:=\sum_{i \in S} \rho_{i}$.

First take $\theta=-\lambda-\rho-\rho_{S}$, where $S:=\left\{0 \leqslant i \leqslant n: \lambda\left(\alpha_{i}^{\vee}\right)<-1\right\}$. Clearly $\theta$ is dominant and moreover $W_{\lambda+\rho}=W_{\lambda+\rho+\theta}$. So, by the above proposition, we get

$$
[M(w * \lambda), L(v * \lambda)]=\left[M\left(w *\left(-\rho-\rho_{S}\right)\right), L\left(v *\left(-\rho-\rho_{S}\right)\right)\right]
$$

Further we write $M(w *(-2 \rho))=\sum_{u} n_{u} L(u *(-2 \rho))$, for some $n_{u} \in \mathbb{Z}^{+}$, and apply the functor $T(-2 \rho, \theta)$ for $\theta:=\sum_{i \in\{0, \ldots, n\} \backslash s} \rho_{i}$. Then, by the above proposition, $M\left(w *\left(-\rho-\rho_{S}\right)\right)=\sum n_{u} L\left(u *\left(-\rho-\rho_{S}\right)\right)$, where the sum runs over only the shortest coset representatives in $W / W_{\rho_{S}}$. This, in particular, gives

$$
\begin{aligned}
& {\left[M\left(w *\left(-\rho-\rho_{S}\right)\right), L\left(v *\left(-\rho-\rho_{S}\right)\right)\right]} \\
& \quad=n_{v}=[M(w *(-2 \rho)), L(v *(-2 \rho))],
\end{aligned}
$$

since, by assumption, $v$ is the shortest element in its coset $v W_{\lambda+\rho}$ and $W_{i+\rho}=W_{\rho_{s}}$. Combining $\left(I_{9}\right)$ and $\left(I_{10}\right)$, we get the corollary.

(1.11) Remark. An appropriate analogue of Proposition (1.7) for $\lambda$ not necessarily integral can easily be proved. But we do not need it.

\section{Reduction of Lusztig's Conjecture to KL-Conjecture}

(2.1) Definition. Let $\mathfrak{g}$ be a finite dimensional simple Lie algebra over $\mathbb{C}$, and let $\stackrel{\mathrm{g}}{ }^{\vee}$ be the (simple) Lie algebra corresponding to the dual root system. Consider the corresponding (untwisted) affine Kac-Moody Lie algebra $\hat{L}\left(\dot{\mathfrak{g}}^{\vee}\right)$ (cf. Section 1.2) with generalized Cartan matrix $A$, and let $\mathrm{g}:=\left(\hat{L}\left(\dot{g}^{\vee}\right)\right)^{\vee}$ be the affine Kac-Moody Lie algebra associated to the generalized Cartan matrix $A^{t}$ (where $A^{t}$ is the transpose of $A$ ). We tentatively call the affine Kac-Moody Lie algebra $\mathfrak{g}$ to be bidually associated

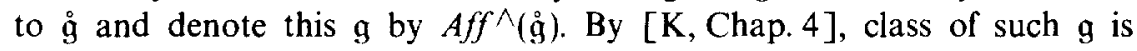


precisely (in the notation of loc. cit.) $A_{l}^{(1)}(l \geqslant 1), D_{l}^{(1)}(l \geqslant 4), E_{6}^{(1)}, E_{7}^{(1)}$, $E_{8}^{(1)}, A_{2 l-1}^{(2)}(l \geqslant 3), D_{l+1}^{(2)}(l \geqslant 2), E_{6}^{(2)}$, and $D_{4}^{(3)}$.

From now on, we fix once and for all such $a \mathfrak{g}=A f f^{\wedge}(\mathfrak{g})$ and follow the notation as in Section 1.1 .

Define the following spaces:

$$
\begin{aligned}
\mathfrak{h}^{*} & =\sum_{i=1}^{n} \mathbb{C} \alpha_{i} \subset \mathfrak{h}^{*}, \\
\mathfrak{h}_{\mathbb{R}}^{*} & =\sum_{i=1}^{n} \mathbb{R} \alpha_{i} \subset \mathfrak{h}^{*}, \\
\grave{\mathfrak{h}} & =\sum_{i=1}^{n} \mathbb{C} \alpha_{i}^{\vee} \subset \mathfrak{h}, \quad \text { and } \\
\mathfrak{}_{\mathbb{R}} & =\sum_{i=1}^{n} \mathbb{R} \alpha_{i}^{\vee} \subset \mathfrak{h} .
\end{aligned}
$$

Then we have orthogonal direct sum decompositions (with respect to the Killing form) (cf. [K, Chap. 6]):

$$
\begin{aligned}
\mathfrak{h} & =\mathfrak{h} \oplus(\mathbb{C} c \oplus \mathbb{C} d), \quad \text { where } c, d \text { are as in Section } 1.1, \text { and } \\
\mathfrak{h}^{*} & =\mathfrak{h}^{*} \oplus\left(\mathbb{C} \delta \oplus \mathbb{C} A_{0}\right), \quad \text { where } \delta \text { is as in Section 1.1, }
\end{aligned}
$$

and $\Lambda_{0} \in \mathfrak{h}^{*}$ is defined as $\Lambda_{0}\left(\alpha_{i}^{\vee}\right)=\delta_{0, i}$, for $0 \leqslant i \leqslant n$, and $\Lambda_{0}(d)=0$.

Observe that (by $\left[\mathrm{K}\right.$, Chap. 6]) $\alpha_{i}(\mathbb{C} c+\mathbb{C} d)=0$, for all $1 \leqslant i \leqslant n$, and hence $\mathfrak{h}^{*}$ is canonically identified with $\operatorname{Hom}_{\mathfrak{C}}(\mathfrak{h}, \mathbb{C})$.

Define $R=R \cap \mathfrak{h}^{*}$, where $R$ is the set of all the roots of $\mathrm{g}$. Then $R^{\circ}$ is the root system for the Lie algebra $\stackrel{\mathrm{g}}{\mathrm{g}}$. Let $\underset{W}{W}$ be the subgroup of the Weyl group $W$ (of $g$ ) generated by the simple reflections $\left\{s_{1}, \ldots, s_{n}\right\}$ (cf. Sect. 1.1).

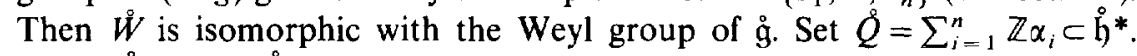
Then $\mathscr{W}$ keeps $Q \subset \mathfrak{h}^{*}$ stable. In particular, we can form the semi-direct product $\mathscr{W} X \mathscr{Q}$.

We recall the following lemma from [K, Prop. 6.5]:

(2.2) Lemma. There is a canonical isomorphism $W \approx W X Q$.

In particular, since $\mathscr{W} X \dot{Q}$ acts on $\phi$ (or $\mathfrak{h}_{\mathbb{R}}^{*}$ ) by affine transformations, we get an affine action of $W$ on $\mathfrak{h}_{\mathbb{R}}^{*}$.

(2.3) Fundamental Alcove. Let $\theta$ be the highest short root (i.e., highest among the short roots) of $\mathfrak{g}$, and hence $\theta^{\vee}$ is the highest coroot for the root system of $\mathfrak{g}$. Write

$$
\theta^{\vee}=\sum_{i=1}^{n} m_{i} \alpha_{i}^{\vee}
$$


Then, by [K, Section 6.4], the number $1+\sum_{i=1}^{n} m_{i}$ is nothing but the dual Coxeter number $h$ of $g$ (cf. Section 1.1).

Fix an integer $l \geqslant 1$. As in [L, Sect. 2.4], define

$$
A_{l}=\left\{\chi \in \mathfrak{h}_{\mathbb{B}}^{*}: \chi\left(\alpha_{i}^{\vee}\right) \leqslant-1, \text { for all } 1 \leqslant i \leqslant n, \text { and } \sum_{i=1}^{n} m_{i} \chi\left(\alpha_{i}^{\vee}\right) \geqslant-l-h+1\right\} .
$$

We also define

$$
\Delta_{d}(\mathbb{Z})=\left\{\chi \in A_{i}: \chi\left(\alpha_{i}^{\vee}\right) \in \mathbb{Z}, \text { for all } 1 \leqslant i \leqslant n\right\} .
$$

Consider the group embedding $\varphi_{l}: W \approx W \mathscr{W} X \dot{Q} \rightarrow W$, defined by

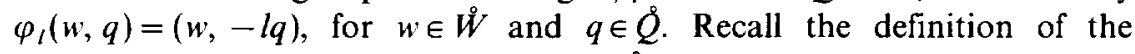
representation $j_{l}: W \rightarrow$ Aff $E$, where $E:=\mathfrak{h}_{R}^{*}$, from [L, Sect. 2.4]. Then we have the following:

(2.4) Lemma. For any $w \in W, \chi \in E$, and $l \geqslant 1$

$$
j_{l}(w) \cdot \chi=\varphi_{l}(w) * \chi
$$

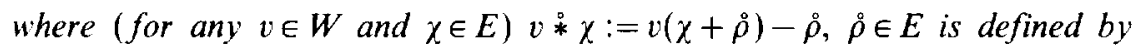
$\dot{\rho}\left(\alpha_{i}^{v}\right)=1$ for all $1 \leqslant i \leqslant n$, and $v(\chi+\stackrel{\rho}{)}$ denotes the action of $W$ on $E$ as given in Lemma (2.2).

Proof. The linear part of the affine map $\varphi_{l}(w)^{*}: E \rightarrow E$ clearly preserves the Killing form. So it suffices to show that the fixed hyperplane $F_{i}$ of $j_{l}\left(s_{i}\right)$ is the same as the fixed hyperplane $\widetilde{F}_{i}$ of $\varphi_{l}\left(s_{i}\right) \stackrel{*}{*}$, for all the simple reflections $s_{i}(0 \leqslant i \leqslant n)$ :

For $1 \leqslant i \leqslant n, \widetilde{F}_{i}=\left\{\chi \in \mathfrak{h}_{\mathbb{R}}^{*}: \chi+\stackrel{\rho}{\rho}\left(\alpha_{i}^{\vee}\right)=0\right\}$,

and by $\left[\mathrm{K}\right.$, Sect. 6.4 and 6.6], $\tilde{F}_{0}=\left\{\chi \in \mathfrak{h}_{\mathbb{R}}^{*}: \chi+\stackrel{\rho}{\rho}\left(\theta^{\vee}\right)=-l\right\}$,

where $\theta^{\vee}$ is the highest coroot as in Section 2.3. But

$$
\chi+\stackrel{\rho}{\rho}\left(\theta^{\vee}\right)=h-1+\sum_{i=1}^{n} m_{i} \chi\left(\alpha_{i}^{\vee}\right), \quad \text { by }\left(\mathrm{I}_{11}\right) .
$$

But then $F_{i}(0 \leqslant i \leqslant n)$, by definition, is the same as $\tilde{F}_{i}$. This proves the lemma.

For any integer $l \geqslant 1$ and $\chi \in \mathfrak{h}^{*}$, define $\theta_{l}(\chi) \in\left(\mathfrak{h}^{1}\right)^{*}$, where $\mathfrak{h}^{1}:=\mathfrak{h} \oplus \mathbb{C} c$, as follows:

$$
\theta_{i}(\chi)\left(\alpha_{i}^{\vee}\right)=\chi\left(\alpha_{i}^{\vee}\right), \quad \text { for all } 1 \leqslant i \leqslant n, \quad \text { and } \quad \theta_{i}(\chi)(c)=-l-h .
$$


Observe that by $[\mathrm{K}$, Section 6.4$]$ and $\left(\mathrm{I}_{11}\right)$,

$$
c=\alpha_{0}^{\vee}+\sum_{i=1}^{n} m_{i} \alpha_{i}^{\vee}, \quad \text { where } m_{i}^{\prime} \text { s are as in }\left(\mathrm{I}_{11}\right) . \quad\left(\mathrm{I}_{12}\right)
$$

(2.5) Lemma. For any $\chi \in \mathfrak{h}_{\mathfrak{s}}^{*}, w \in W$, and $l \geqslant 1$,

$$
\theta_{l}\left(\left(\varphi_{l} w\right) * \chi \chi\right)=w * \theta_{l}(\chi), \quad \text { as elements of }\left(\mathbf{h}^{1}\right)^{*}, \quad\left(\mathrm{I}_{13}\right)
$$

where $*$ is as in Lemma (2.4), and the action of $w *$ on $\mathfrak{h}^{*}$ (as given in $\mathrm{I}_{1}$ ) gives rise to an action on $\left(\mathfrak{h}^{1}\right)^{*}$.

Proof. Both the sides of $\left(\mathrm{I}_{13}\right)$ take the same value on $c$ (see [K, Identity 6.2 .5 on p. 82]), so it suffices to show that they take the same value on $\stackrel{\mathrm{h}}{\mathrm{i}}$

If $w \in \mathscr{W}$, it is clearly true. So take $\alpha \in \mathscr{Q}$ (cf. Lemma 2.2), and consider the translation $w=t_{\alpha}$ by $\alpha$. Then

$$
\text { left } \text { side }_{\mid \mathfrak{h}}=\chi-l \alpha, \quad \text { whereas }
$$

right side $\operatorname{l\mathfrak {h}}_{\mid \mathfrak{h}}=\left[t_{\alpha}\left(\theta_{l}(\chi)+\rho\right)-\rho\right]_{\mid \mathfrak{h}}$

$$
\begin{array}{lll}
=\left[\theta_{l}(\chi)+\left\langle\theta_{l}(\chi)+\rho, c\right\rangle \alpha\right]_{i h}, & & \text { by }[\mathrm{K}, \text { Identity } 6.5 .2, \mathrm{p} .87] \\
=\chi+(-l-h+h) \alpha, & & \text { by }\left(\mathrm{I}_{12}\right) \\
=\chi-l \alpha . &
\end{array}
$$

For any integer $l \geqslant 1$, and affine Kac-Moody Lie algebra $\mathfrak{g}$, define

$$
\begin{gathered}
H_{l}=\left\{\lambda \in\left(\mathfrak{h}^{1}\right)^{*}:\left\langle\lambda, \alpha_{i}^{\vee}\right\rangle \text { is real and } \leqslant-1, \text { for all } 0 \leqslant i \leqslant n,\right. \\
\text { and moreover } \lambda(c)=-l-h\}, \quad \text { and } \\
H_{l}(\mathbb{Z})=\left\{\lambda \in H_{l}:\left\langle\lambda, \alpha_{i}^{\vee}\right\rangle \in \mathbb{Z}, \text { for all } 0 \leqslant i \leqslant n\right\} .
\end{gathered}
$$

We have the following simple

(2.6) Lemma. For any $l \geqslant 1$,

$$
\theta_{l}\left(\Delta_{l}\right)=H_{l}, \quad \text { and moreover } \quad \theta_{l}\left(\Delta_{l}(\mathbb{Z})\right)=H_{l}(\mathbb{Z})
$$

where $A_{l}, A_{l}(\mathbb{Z})$ are as in Section 2.3 . 
Proof. By definition

$$
\begin{aligned}
& \theta_{l}(\chi)(c)=-l-h, \quad \text { i.e., by }\left(\mathrm{I}_{12}\right), \\
& \theta_{l}(\chi)\left(\alpha_{0}^{\vee}+\sum_{i=1}^{n} m_{i} \alpha_{i}^{\vee}\right)=-l-h .
\end{aligned}
$$

Hence

$$
\theta_{l}(\chi)\left(\alpha_{0}^{\vee}\right)=-l-h-\sum_{i=1}^{n} m_{i} \chi\left(\alpha_{i}^{\vee}\right) \leqslant-l-h+l+h-1, \quad \text { for } \quad \chi \in \Delta_{l} .
$$

Let us recall the following conjecture due to Lusztig [ $\mathrm{L}$, Conjecture 2.5 (c)]. (Actually he wrote down the conjecture only for simply-laced $\mathfrak{g}$, in which case $\mathfrak{g}$ as below is nothing but $\hat{L}(\stackrel{\mathfrak{g}}{)})$, cf. Section 1.2.)

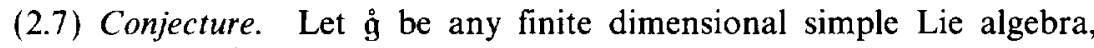
and let $\mathfrak{g}:=A f f^{\wedge}(\mathfrak{g})$ (cf. Section 2.1). Fix an integer $l \geqslant 1$. Then for any $\chi \in \Delta_{l}(\mathbb{Z})$ (cf. Section 2.3), and $w \in W$ (where $W$ is the Weyl group associated to $\mathfrak{g}$ )

$$
L\left(\theta_{l}\left(j_{l}(w) \cdot \chi\right)\right)=\sum_{\substack{v \leq w^{\prime} \\ v \in W}}(-1)^{\ell(v)+l\left(w^{\prime}\right)} P_{v, w^{\prime}}(1) M\left(\theta_{l}\left(j_{l}(v) \cdot \chi\right)\right),
$$

in the Grothendieck group of $U\left(\mathfrak{g}^{1}\right)$-modules, where $\mathfrak{g}^{1}$ is the commutator subalgebra $[\mathfrak{g}, \mathfrak{g}], \theta_{l}$ (resp. $j_{l}$ ) is as in Section 2.4 (resp. Section 2.3), $M()$ (resp. $L()$ ) is the Verma module (resp. irreducible quotient) as in Section 1.3, $w^{\prime}$ is the (unique) shortest element $\in W$ satisfying $j_{l}\left(w^{\prime}\right) \cdot \chi=$ $j_{l}(w) \cdot \chi$, and $P_{v, w^{\prime}}$ of course is the Kazhdan-Lusztig polynomial for the Coxeter group $W$.

Combining Lemmas 2.4-2.6, we get the following equivalent reformulation of the above conjecture:

(2.8) Proposition. Let the notation and assumptions be as in the above conjecture (2.7). Then its (Conjecture 2.7) validity is equivalent to the validity of the following statement:

For any $\lambda \in H_{l}(\mathbb{Z})$ and $w \in W$

$$
L(w * \lambda)=\sum_{\substack{v \leq w^{\prime} \\ v \in W}}(-1)^{l(v)+l\left(w^{\prime}\right)} P_{v, w^{\prime}}(1) M(v * \lambda),
$$

in the Grothendieck group of $U\left(\mathrm{~g}^{1}\right)$-modules, where $H_{l}(\mathbb{Z})$ is as defined in Section 2.5, $w^{\prime}$ is the (unique) shortest element $\in W$ satisfying $w^{\prime} * \lambda=w * \dot{\lambda}$, and $*$ is the action as in Lemma (2.5). 
Let us recall the following celebrated conjecture due to Kazhdan-Lusztig [KL, Conjecture 1.5]. It was originally conjectured for the finite case, and of course proved independently by Beilinson-Bernstein and Brylinski-Kashiwara. The following is its direct extension to the affine Kac-Moody case, claimed to have been proved recently by Casian [C, Thm. 17]. It may be mentioned that the corresponding conjecture (cf. [DGK, Conjecture 5.16]) for $L(w \rho-\rho)$ has been proved independently by Casian and Kashiwara-Tanisaki.

(2.9) KL-Conjecture for the Affine Algebras. Let $\mathfrak{g}$ be any affine (including twisted affine) Kac-Moody Lie algebra. Then for any $w \in W$ (where $W$ is the Weyl group associated to $\mathrm{g}$ )

$$
L(-w \rho-\rho)=\sum_{v \leqslant w}(-1)^{l(v)+l(w)} P_{v, w}(1) M(-v \rho-\rho),
$$

in the Grothendieck group of $U(\mathfrak{g})$-modules.

Finally we come to the following main result of this paper:

(2.10) THEOREM. Let $\mathfrak{g}:=A f f^{\wedge}(\stackrel{\mathrm{g}}{)})$ be the affine Kac-Moody Lie algebra as in Conjecture (2.7). Then the validity of the KL-conjecture (2.9) for $\mathrm{g}$ implies the validity of Lusztig's conjecture (2.7) for $\mathrm{g}$.

Proof. In view of Proposition (2.8), it suffices to show that the validity of (2.9) for $g$ implies the validity of $\left(I_{14}\right)$ :

Fix any $\lambda \in H_{i}(\mathbb{Z})$ and $w \in W$, and arbitrarily extend $\lambda$ to $\lambda \in \mathfrak{h}^{*}$. Define a subset $S \subset\{0,1, \ldots, n\}$ by $S=\left\{i: \lambda\left(\alpha_{i}^{\vee}\right)<-1\right\}$, and take $\theta=-\bar{\lambda}-\rho-\rho_{S}$ (cf. proof of Corollary 1.10). Then $\theta$ is clearly dominant integral and, moreover, the isotropy

$$
W_{\bar{\lambda}+\rho}=W_{\bar{\lambda}+\theta+\rho} . \quad\left(\mathrm{I}_{15}\right)
$$

By Conjecture (2.9), write

$$
L\left(w^{\prime} *(-2 \rho)\right)=\sum_{v \leqslant w^{\prime}}(-1)^{l(v)+l\left(w^{\prime}\right)} P_{v, w^{\prime}}(1) M(v *(-2 \rho)), \quad\left(\mathrm{I}_{16}\right)
$$

where $w^{\prime}$ is the shortest element in $w W_{\bar{\lambda}+\rho}$.

Applying the translation functor $T\left(-2 \rho, \rho-\rho_{S}\right)$ to $\left(\mathrm{I}_{16}\right)$ and using Proposition (1.7), we get

$$
L\left(w^{\prime} *\left(-\rho-\rho_{S}\right)\right)=\sum_{v \leqslant w^{\prime}}(-1)^{l(v)+/\left(w^{\prime}\right)} P_{v, w^{\prime}}(1) M\left(v *\left(-\rho-\rho_{S}\right)\right)
$$


Write

$$
L\left(w^{\prime} * \bar{\lambda}\right)=\sum_{\bar{v} \in W / W_{\bar{i}+\rho}} n_{\bar{v}} M(\bar{v} * \bar{\lambda}), \quad \text { for some } n_{\bar{v}} \in \mathbb{Z} . \quad\left(\mathrm{I}_{18}\right)
$$

Applying the functor $T\left(\bar{\lambda}, \theta=-\bar{\lambda}-\rho-\rho_{S}\right)$ to $\left(\mathrm{I}_{18}\right)$, we get (by Proposition 1.7),

$$
L\left(w^{\prime} *\left(-\rho-\rho_{S}\right)\right)=\sum_{\bar{v} \in W_{i} \boldsymbol{W}_{i+\rho}} n_{\bar{i}} M\left(\bar{v} *\left(-\rho-\rho_{S}\right)\right) \quad\left(\text { use } \mathrm{I}_{15}\right) . \quad\left(\mathrm{I}_{19}\right)
$$

Comparing $\mathrm{I}_{17}-\mathrm{I}_{19}$ together with $\mathrm{I}_{15}$ (and then considering the $\mathrm{g}$-modules involved, just as $\mathbf{g}^{1}$-modules) proves the theorem. (Observe that $W_{i_{+} \rho}=$ $\left\{w \in W: w\left(\lambda+\rho_{\mid \mathfrak{b}^{1}}\right)=\lambda+\rho_{\mid \mathrm{b}^{1}}\right\}$, as the right hand subgroup of $W$ is also generated by the simple reflections it contains, cf. [K, Proof of Proposition 3.12].)

Remark. This is a slightly revised version of the preprint, distributed in May 1990, with the title "Proof of Lusztig's Conjecture Concerning Negative Level Representations of Affine Lie Algebras."

\section{ACKNOWLEDGMENTS}

I thank G. Lusztig for his comments on an earlier draft of this paper, and L. Scott for his suggestions regarding the exposition.

\section{REFERENCES}

[B] N. Bourbaki, "Groupes et Algèbres de Lie," Chap. IV-VI, Hermann, Paris, 1968.

[C] L. CASIAN, Kazhdan-Lusztig conjecture in the negative level case (Kac-Moody algebras of affine type), preprint.

[DGK] V. V. DeOdhar, O. GabBer, AND V. KaC, Structure of some categories of representations of infinite-dimensional Lie algebras, Adv. Math. 45 (1982), 92-116.

[K] V. G. KAC, "Infinite Dimensional Lie Algebras," Third edition, Cambridge University Press, Cambridge, 1990.

[KL] D. Kazhdan and G. LuszTig, Representations of Coxeter groups and Hecke algebras, Invent. Math. 53 (1979), 165-184.

[Ku] S. Kumar, Extension of the category $\mathcal{O}^{g}$ and a vanishing theorem for the Ext functor for Kac-Moody algebras, J. Algebra 108 (1987), 472-491.

[L] G. LusztiG, On quantum groups, J. Algebra 131 (1990), 466-475. 